\title{
Systematically comparing COVID-19 with the 2009 influenza pandemic for hospitalized patients
}

\author{
Pengfei Li ${ }^{\mathrm{a}}$, Yining Wang ${ }^{\mathrm{a}}$, Maikel P. Peppelenbosch ${ }^{\mathrm{a}}$, Zhongren Ma ${ }^{\mathrm{b}, *}$, Qiuwei Pan ${ }^{\mathrm{a}, \mathrm{b}, *}$ \\ a Department of Gastroenterology and Hepatology, Erasmus MC-University Medical Center, Rotterdam, The Netherlands \\ b Biomedical Research Center, Northwest Minzu University, Lanzhou, China
}

\section{A R T I C L E I N F O}

\section{Article history:}

Received 27 July 2020

Received in revised form 4 November 2020

Accepted 5 November 2020

\section{Keywords:}

COVID-19

2009 influenza pandemic

Clinical features

Symptoms

Comorbidities

Meta-analysis

\begin{abstract}
A B S T R A C T
Objectives: This study aimed to comprehensively compare the clinical features of hospitalized COVID-19 patients with hospitalized 2009 influenza pandemic patients.

Methods: Medline, Embase, Web of Science, Cochrane CENTRAL, and Google scholar were systematically searched to identify studies related to COVID-19 and the 2009 influenza pandemic. The pooled incidence rates of clinical features were estimated using the DerSimonian-Laird random-effects model with the Freeman-Tukey double arcsine transformation method.

Results: The incidence rates of fever, cough, shortness of breath, sore throat, rhinorrhea, myalgia/muscle pain, or vomiting were found to be significantly higher in influenza patients when compared with COVID19 patients. The incidence rates of comorbidities, including cardiovascular disease/hypertension and diabetes, were significantly higher in COVID-19 compared with influenza patients. In contrast, comorbidities such as asthma, chronic obstructive pulmonary disease, and immunocompromised conditions were significantly more common in influenza compared with COVID-19 patients. Unexpectedly, the estimated rates of intensive care unit admission, treatment with extracorporeal membrane oxygenation, treatment with antibiotics, and fatality were comparable between hospitalized COVID-19 and 2009 influenza pandemic patients.

Conclusions: This study comprehensively estimated the differences and similarities of the clinical features and burdens of hospitalized COVID-19 and 2009 influenza pandemic patients. This information will be important to better understand the current COVID-19 pandemic.

(c) 2020 The Author(s). Published by Elsevier Ltd on behalf of International Society for Infectious Diseases.

This is an open access article under the CC BY license (http://creativecommons.org/licenses/by/4.0/).
\end{abstract}

\section{Introduction}

The ongoing COVID-19 pandemic is spreading unprecedentedly, and the eventual outcome of this pandemic remains largely uncertain. The causative agent of COVID-19 - severe acute respiratory syndrome coronavirus-2 (SARS-CoV-2) - is highly contagious (Rothan and Byrareddy, 2020). The clinical manifestations of SARS-CoV-2 infection range from asymptomatic to severe pneumonia, with some cases resulting in multiple organ failure or death (Adhikari et al., 2020). The main reported symptoms include fever, cough, shortness of breath, myalgia, and fatigue (Huang et al., 2020). Death cases have primarily been elderly people with comorbidities such as hypertension, coronary heart disease and diabetes (Adhikari et al., 2020).

\footnotetext{
* Corresponding authors at: Biomedical Research Center, Northwest Minzu University, No. 1, Xibei Xincun, Lanzhou, 730030, China.

E-mail addresses: mzr@xbmu.edu.cn (Z. Ma), q.pan@erasmusmc.nl (Q. Pan).
}

It has widely been recognized that SARS-CoV-2 and influenza virus infections share substantial similarities in viral shedding, transmission dynamics and clinical features of respiratory illnesses (He et al., 2020; Petersen et al., 2020). Recent studies have attempted to revisit the historical experience of 1918 and 2009 influenza pandemics, in order to learn lessons for better understanding the current COVID-19 pandemic. These recent studies have revealed similarities and differences in transmissibility, epidemic wave, and mortality rate between COVID-19 and influenza pandemics (He et al., 2020; Petersen et al., 2020; Petrosillo et al., 2020). It is believed that the published studies on comparison between influenza and COVID-19 pandemics are mainly focused on epidemiological features in the general population (Petersen et al., 2020; Viboud and Simonsen, 2012). In fact, the disease burden of both COVID-19 and influenza pandemics largely lies in the hospitalized patient population, which has not been systematically and comparatively studied.

There is a scarcity of clinical data available on the 1918 influenza pandemic, but the latest influenza pandemic in 2009 has been extensively studied. The current study performed a systematic 
review and meta-analysis to comprehensively compare the burden and key clinical features of hospitalized patients between the 2009 influenza and COVID-19 pandemic.

\section{Methods}

\section{Data sources and search strategies}

A systematic search was conducted in Medline, Embase, Web of Science, Cochrane CENTRAL, and Google scholar. Databases were searched for articles in English language from inception until 2030. All searches from databases were performed by a biomedical information specialist from the medical library. An exhaustive set of search terms was used to identify studies providing clinical features/outcomes of hospitalized patients for the $2009 \mathrm{H} 1 \mathrm{~N} 1$ influenza or COVID-19 pandemic. The full search strategies are provided in the Supplementary file S1. This study was performed in accordance with the Preferred Reporting Items for Systematic Reviews and Meta-Analysis (PRISMA) guidelines.

\section{Selection criteria}

Studies were included if they met the following criteria: i) they contained clinical characteristics of SARS-CoV-2-confirmed patients and ii) they contained clinical characteristics of 2009 pandemic H1N1 influenza-confirmed patients. Studies were excluded if they met following criteria: i) they belonged to systematic review, meta-analysis, case reports, perspectives, or conference abstracts; ii) they were animal coronavirus studies; iii) they had no primary data or incomplete data; iv) they had duplicate data; and v) they were of low quality. Detailed full-text review and data extraction were independently performed by two investigators: PL and YW. The investigators resolved discrepancies by jointly reviewing the study in question. If no consensus was reached, another reviewer $(\mathrm{QP})$ functioned as an arbiter to resolve disagreements.

\section{Data extraction and quality assessment}

Eligible studies were further screened to extract data. In cases of multiple studies involving the same population, the most comprehensive or most recent one was included. The quality of studies was assessed using the Joanna Briggs Institute checklist for prevalence/incidence studies, which enabled assessment of included studies in relation to risk of bias, rigour, and transparency (Munn et al., 2020). Studies scoring 1-3 were defined as lowquality, 4-6 as average-quality, and 7-9 as high-quality (Supplementary data Table 1 ).

\section{Statistics analysis}

After checking for consistency, the Metaprop module in the R3.6.3 statistical software package (Version 3.6.3 for Windows, Foundation for Statistical Computing, Vienna, Austria) was used for meta-analysis. A 95\% confidence interval (95\% CI) was estimated using the Wilson score method, and the pooled incidence rate was calculated by the DerSimonian-Laird random-effects model with the Freeman-Tukey double arcsine transformation method. Statistical heterogeneity was assessed using the Cochran Q statistics and $\mathrm{I}^{2}$ statistics, with $\mathrm{I}^{2}$ statistics categorized as low $\left(\mathrm{I}^{2}\right.$ $=25-50 \%)$, moderate $\left(\mathrm{I}^{2}=50-75 \%\right)$, high $\left(\mathrm{I}^{2}>75 \%\right)$, or no statistical heterogeneity $\left(\mathrm{I}^{2}=0 \%\right)$. A random-effects model was used when the heterogeneity was $>50 \%$. The Mann-Whitney test (nonparametric test) was used to compare the COVID-19 and 2009 influenza pandemic patient groups. A $p$-value $<0.05$ was considered as significant.

\section{Results}

Study and patient characteristics

The database search yielded 21,986 records after excluding replications. A total of 113 studies with 36,422 hospitalized COVID19 patients and 84 studies with 23,167 hospitalized influenza patients were finally included (Figure 1 ). Patients from the two pandemics had similar sex distributions, with slightly $>50 \%$ being male. The average age of hospitalized COVID-19 patients was 52 years ( $S D \pm 16.93)$, which was much older than the influenza patients (average 26 years, $\mathrm{SD} \pm 15.57$ ) (Table 1 ).

Comparative analysis of symptoms and comorbidities between hospitalized COVID-19 and influenza patients

The highest prevalent symptom in hospitalized COVID-19 patients was fever $\left(72.08 \%, 95 \%\right.$ CI $\left.63.27-80.13 ; I^{2}=99 \%\right)$, followed by cough $\left(57.99 \%, 95 \%\right.$ CI $\left.53.32-62.59 ; I^{2}=97 \%\right)$, shortness of breath (32.89\%, 95\% CI 27.09-38.95; $\left.I^{2}=98 \%\right)$, fatigue $(30.20 \%, 95 \%$ CI $\left.24.75-35.93 ; I^{2}=96 \%\right)$, myalgia/muscle pain $(18.97 \%$, 95\% CI $14.23-$ $\left.24.20 ; I^{2}=98 \%\right)$, diarrhea $\left(10.16 \%, 95 \%\right.$ CI $\left.7.23-13.51 ; I^{2}=97 \%\right)$, sore throat $\left(9.48 \%, 95 \%\right.$ CI $\left.7.47-11.69 ; I^{2}=88 \%\right)$, vomiting/nausea $(8.67 \%$, $95 \%$ CI $\left.5.40-12.59 ; I^{2}=98 \%\right)$, and rhinorrhea $(8.48 \%$, 95\% CI $6.02-$ $11.28 ; I^{2}=92 \%$ ) (Figure 2, Supplementary S2-10). Fever and cough were the most common symptoms in hospitalized 2009 influenza patients, with incidence rates of $89.99 \%$ (95\% CI 87.64-92.13; $I^{2}=9$ $6 \%$ ) and $85.31 \%$ (95\% CI 82.84-87.63; $\left.I^{2}=95 \%\right)$, respectively, followed by shortness of breath $\left(49.19 \%, 95 \%\right.$ CI $40.40-58.01 ; I^{2}=$ $98 \%)$, fatigue (48.20\%, 95\% CI 29.64-67.03; $\left.I^{2}=99 \%\right)$, rhinorrhea

Table 1

Characteristics, management and outcome of hospitalized COVID-19 and 2009 influenza patients.

\begin{tabular}{|c|c|c|c|c|c|c|c|}
\hline \multirow[b]{2}{*}{ Characteristics } & \multicolumn{3}{|l|}{ COVID-19 pandemic } & \multicolumn{4}{|c|}{2009 influenza pandemic } \\
\hline & $\begin{array}{l}\text { Estimates } \\
(95 \% \mathrm{CI})\end{array}$ & Study No. & Patient No. & $\begin{array}{l}\text { Estimates } \\
(95 \% \mathrm{CI})\end{array}$ & Study No. & Patient No. & $p$-value \\
\hline Sex $($ male $\%)$ & $20,425(56.08 \%)$ & 113 & 36,422 & $12,556(54.20 \%)$ & 84 & 23,167 & 1 \\
\hline Age, years (mean $\pm S D$, range) & $52.11 \pm 16.93(0.2-96)$ & 37 & 5085 & $26.27 \pm 15.57(0-94)$ & 47 & 12,347 & i \\
\hline \multicolumn{8}{|l|}{ Management } \\
\hline Antibiotics use & $67.44 \%(51.91-81.27)$ & 23 & 5350 & $60.45 \%(47.93-72.31)$ & 13 & 2569 & 0.3081 \\
\hline Mechanical ventilation & $27.10 \%(20.91-33.75)$ & 46 & 17,101 & $14.70 \%(6.65-25.12)$ & 13 & 4975 & 0.0603 \\
\hline ECMO & $3.10 \%(1.45-5.25)$ & 19 & 6644 & $5.79 \%$ & 2 & 863 & 1 \\
\hline ICU admission & $17.74 \%(13.93-21.89)$ & 35 & 15,636 & $16.03 \%(11.55-21.06)$ & 36 & 9083 & 0.4416 \\
\hline \multicolumn{8}{|l|}{ Outcome } \\
\hline Death & $12.94 \%(10.93-15.10)$ & 54 & 25,390 & $9.63 \%(6.05-13.90)$ & 28 & 5075 & 0.1682 \\
\hline
\end{tabular}

Abbreviations: ECMO, extracorporeal membrane oxygenation; ICU, intensive care unit. 


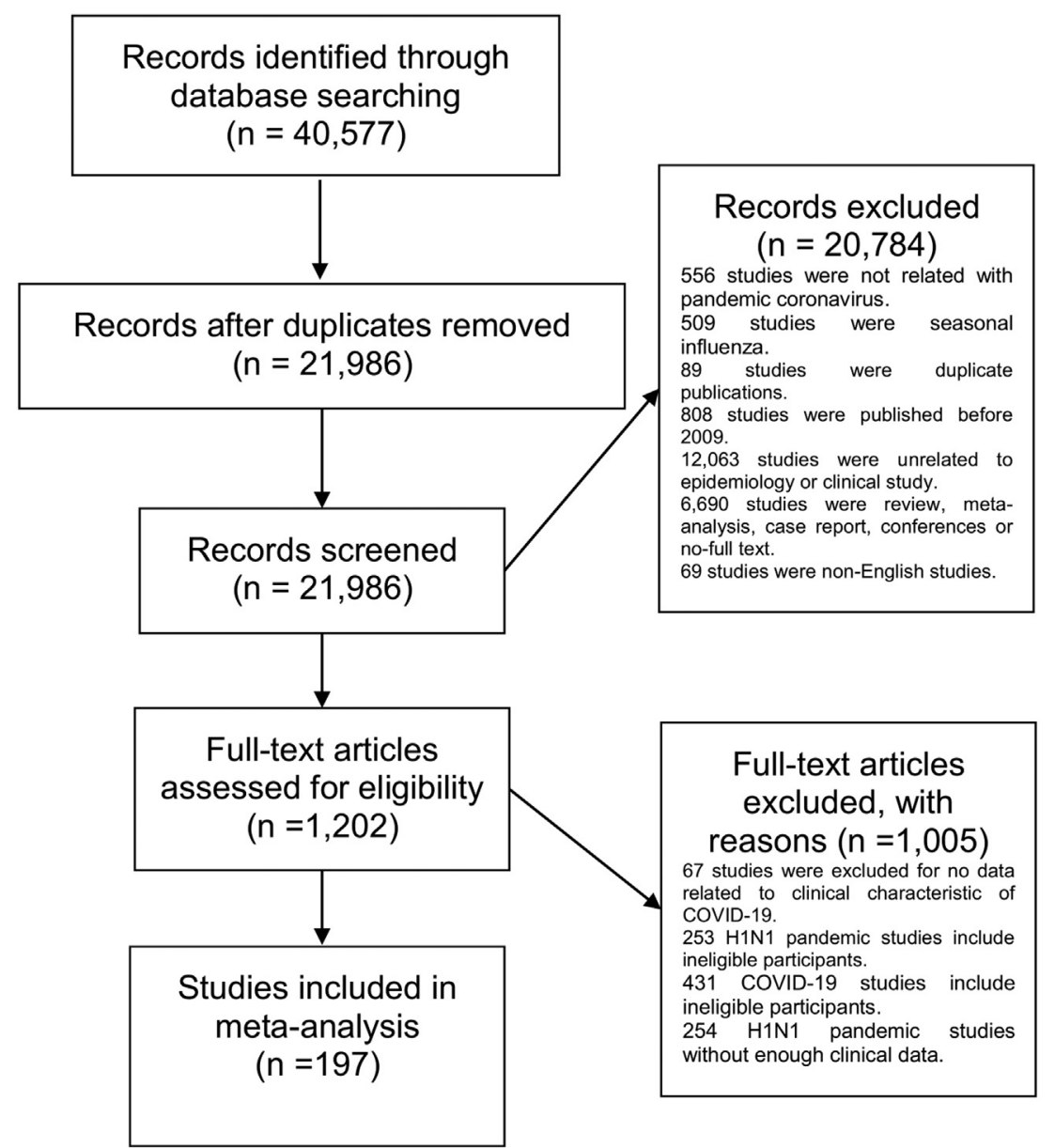

Figure 1. Study selection.

Symptom
COVID-19 patients
Fever
Cough
Shortness of breath
Fatigue
Myalgia/Muscle pain
Diarrhea
Sore throat
Vomiting/Nausea
Rhinorrhoea
H1N1 influenza patients
Fever
Cough
Shortness of breath
Fatigue
Rhinorrhoea
Sore throat
Myalgia/Muscle pain
Vomiting/Nausea
Diarrhea

$\begin{array}{rrcr}\text { Events } & \text { Total } & \mathbf{I}^{2} & \text { Proportion (95\% Cl) } \\ 10674 & 16006 & 99 \% & 72.08 \%(63.27-80.13) \\ 8776 & 14717 & 97 \% & 57.99 \%(53.32-62.59) \\ 5615 & 14211 & 98 \% & 32.89 \%(27.09-38.95) \\ 2059 & 6875 & 96 \% & 30.20 \%(24.75-35.93) \\ 2297 & 12865 & 98 \% & 18.97 \%(14.23-24.20) \\ 1576 & 14434 & 97 \% & 10.16 \%(7.23-13.51) \\ 813 & 8502 & 88 \% & 9.48 \%(7.47-11.69) \\ 1137 & 13174 & 98 \% & 8.67 \%(5.40-12.59) \\ 544 & 8040 & 92 \% & 8.48 \%(6.02-11.28) \\ & & & \\ 17762 & 20571 & 96 \% & 89.99 \%(87.64-92.13) \\ 17657 & 20710 & 95 \% & 85.31 \%(82.84-87.63) \\ 2568 & 5521 & 98 \% & 49.19 \%(40.40-58.01) \\ 1570 & 4391 & 99 \% & 48.20 \%(29.64-67.03) \\ 7561 & 15997 & 98 \% & 38.57 \%(33.11-44.17) \\ 7144 & 17268 & 98 \% & 37.28 \%(32.00-42.70) \\ 4564 & 16940 & 98 \% & 30.12 \%(24.68-35.84) \\ 3214 & 12846 & 96 \% & 24.27 \%(20.51-28.25) \\ 2321 & 17433 & 91 \% & 11.27 \%(9.63-13.01)\end{array}$

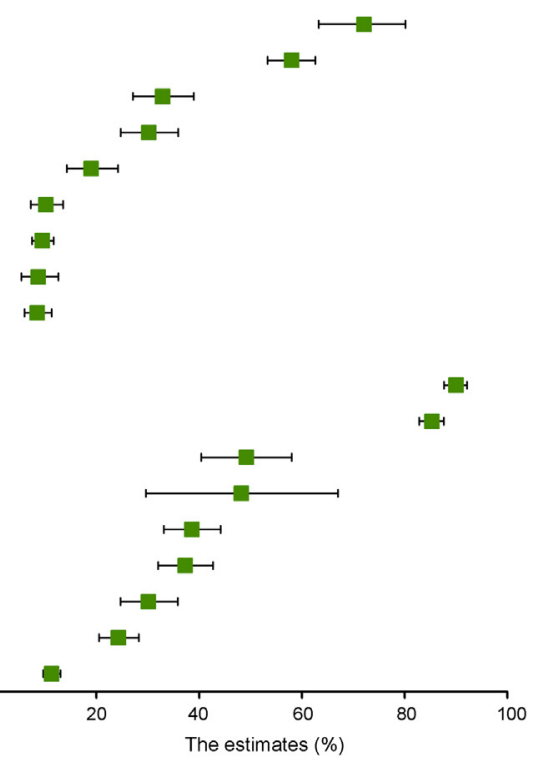

Figure 2. Forest plot of incidence rate of common symptoms in hospitalized COVID-19 and 2009 influenza pandemic patients. 
Table 2

Comparative analysis of symptoms and comorbidities in hospitalized COVID-19 and 2009 influenza patients.

\begin{tabular}{|c|c|c|c|c|c|c|c|}
\hline \multirow[b]{2}{*}{ Characteristics } & \multicolumn{3}{|l|}{ COVID-19 pandemic } & \multicolumn{4}{|c|}{2009 influenza pandemic } \\
\hline & Estimates $(95 \% \mathrm{CI})$ & Study No. & Patient No. & $\begin{array}{l}\text { Estimates } \\
(95 \% \mathrm{CI})\end{array}$ & Study No. & Patient No. & $p$-value \\
\hline \multicolumn{8}{|l|}{ Symptoms } \\
\hline Cough & $57.99 \%(53.32-62.59)$ & 56 & 14,717 & $85.31 \%(82.84-87.63)$ & 82 & 20,710 & $<0.0001$ \\
\hline Fever & $72.08 \%(63.27-80.13)$ & 57 & 16,006 & $89.99 \%(87.64-92.13)$ & 78 & 20,571 & $<\mathbf{0 . 0 0 0 1}$ \\
\hline Diarrhea & $10.16 \%(7.23-13.51)$ & 41 & 14,434 & $11.27 \%(9.63-13.01)$ & 63 & 17,433 & 0.3897 \\
\hline Fatigue & $30.20 \%(24.75-35.93)$ & 34 & 6,875 & $48.20 \%(29.64-67.03)$ & 16 & 4391 & 0.0965 \\
\hline Shortness of breath & $32.89 \%(27.09-38.95)$ & 54 & 14,211 & $49.19 \%(40.40-58.01)$ & 25 & 5521 & 0.0249 \\
\hline Sore throat & $9.48 \%(7.47-11.69)$ & 34 & 8502 & $37.28 \%(32.00-42.70)$ & 61 & 17,268 & $<\mathbf{0 . 0 0 0 1}$ \\
\hline Rhinorrhea & $8.48 \%(6.02-11.28)$ & 27 & 8040 & $38.57 \%(33.11-44.17)$ & 55 & 15,997 & $<\mathbf{0 . 0 0 0 1}$ \\
\hline Myalgia/muscle pain & $18.97 \%(14.23-24.20)$ & 36 & 12,865 & $30.12 \%(24.68-35.84)$ & 68 & 16,940 & 0.0242 \\
\hline Vomiting/nausea & $8.67 \%(5.40-12.59)$ & 27 & 13,174 & $24.27 \%(20.51-28.25)$ & 52 & 12,846 & $<0.0001$ \\
\hline \multicolumn{8}{|l|}{ Comorbidity } \\
\hline Cardiovascular disease/ Hypertension & $28.76 \%(22.91-34.99)$ & 83 & 33,019 & $13.11 \%(10.84-15.55)$ & 41 & 10,144 & $<0.0001$ \\
\hline Obesity & $28.48 \%(19.04-38.94)$ & 18 & 12,819 & $17.89 \%(13.40-22.86)$ & 46 & 13,409 & 0.1081 \\
\hline Diabetes & $16.38 \%(13.65-19.30)$ & 89 & 33,407 & $11.12 \%(9.39-12.97 \%)$ & 58 & 17,031 & 0.0120 \\
\hline Asthma & $8.42 \%(6.69-10.32)$ & 19 & 7732 & $16.09 \%(13.15-19.26)$ & 53 & 16,034 & 0.0033 \\
\hline COPD & $4.93 \%(3.78-6.21)$ & 44 & 14,107 & $9.52 \%(7.09-12.24)$ & 37 & 11,493 & 0.0003 \\
\hline Immunocompromised & $4.39 \%(2.50-6.71)$ & 29 & 9111 & $9.99 \%(8.30-11.82)$ & 48 & 17,521 & $<\mathbf{0 . 0 0 0 1}$ \\
\hline Cancer/malignancy & $4.75 \%(3.59-6.06)$ & 40 & 23,815 & $5.76 \%(4.09-7.67)$ & 25 & 6557 & 0.2906 \\
\hline Heart disease & $12.89 \%(9.71-16.43)$ & 43 & 13,876 & $8.66 \%(6.30-11.34)$ & 28 & 7396 & 0.2249 \\
\hline Renal disease & $8.10 \%(5.93-10.56)$ & 56 & 26,016 & $4.90 \%(3.86-6.05)$ & 35 & 9793 & 0.1676 \\
\hline
\end{tabular}

COPD, chronic obstructive pulmonary disease.

(38.57\%, 95\% CI 33.11-44.17; $\left.I^{2}=98 \%\right)$, sore throat $(37.28 \%, 95 \% \mathrm{CI}$ $\left.32.00-42.70 ; I^{2}=98 \%\right)$, myalgia/muscle pain $(30.12 \%, 95 \%$ CI $24.68-$ $\left.35.84 ; I^{2}=98 \%\right)$, vomiting/nausea $\left(24.27 \%, 95 \%\right.$ CI $20.51-28.25 ; I^{2}=$ $96 \%$ ), and diarrhea (11.27\%, 95\% CI 9.63-13.01; $I^{2}=91 \%$ ) (Figure 2; Supplementary S11-19). Notably, cough (85.31\% vs. 57.99\%), fever (89.99\% vs. $72.08 \%$ ), shortness of breath $(49.19 \%$ vs. $32.89 \%)$, sore throat (37.28\% vs. $9.48 \%$ ), rhinorrhea (38.57\% vs. $8.48 \%$ ), myalgia/ muscle pain (30.12\% vs. $18.97 \%$ ), and vomiting (24.27\% vs. $8.67 \%)$ were significantly higher in influenza than COVID-19 patients $(p<$ 0.05 ; Table 2). It was estimated that $27.60 \%$ (95\% CI 17.55-38.93; $I^{2}$ = 98\%) of $\mathrm{H} 1 \mathrm{~N} 1$ patients presented with pneumonia symptoms (Supplementary S20). However, the included COVID-19 studies provided little information on the rate of pneumonia. A recent study reported that $86.1 \%$ of hospitalized COVID-19 patients were diagnosed with ground-glass opacities in the lungs (Zhao et al., 2020), which is highly related to pneumonia symptoms.

Cardiovascular disease/hypertension was the most common comorbidity in hospitalized COVID-19 patients $(28.76 \%, 95 \% \mathrm{CI}$ $\left.22.91-34.99 ; I^{2}=99 \%\right)$, followed by obesity $(28.48 \%$, 95\% CI $19.04-$ $\left.38.94 ; I^{2}=99 \%\right)$, diabetes ( $16.38 \%, 95 \%$ CI $\left.13.65-19.30 ; I^{2}=98 \%\right)$, heart disease $\left(12.89 \%, 95 \% \mathrm{CI} 9.71-16.43 ; I^{2}=97 \%\right)$, asthma $(8.42 \%$, $95 \%$ CI 6.69-10.32; $\left.I^{2}=80 \%\right)$, and renal disease $(8.10 \%$, 95\% CI 5.93$10.56 ; I^{2}=97 \%$ ) (Figure 3; Supplementary data S21-29). Obesity was the most common comorbidity in influenza patients, with incidence rates of $17.89 \%$ (95\% CI 13.40-22.86; $I^{2}=98 \%$ ), followed by asthma $\left(16.09 \%, 95 \%\right.$ CI $\left.13.15-19.26 ; I^{2}=96 \%\right)$, cardiovascular disease/hypertension (13.11\%, 95\% CI 10.84-15.55; $I^{2}=91 \%$ ), diabetes (11.12\%, 95\% CI 9.39-12.97; $\left.I^{2}=92 \%\right)$,

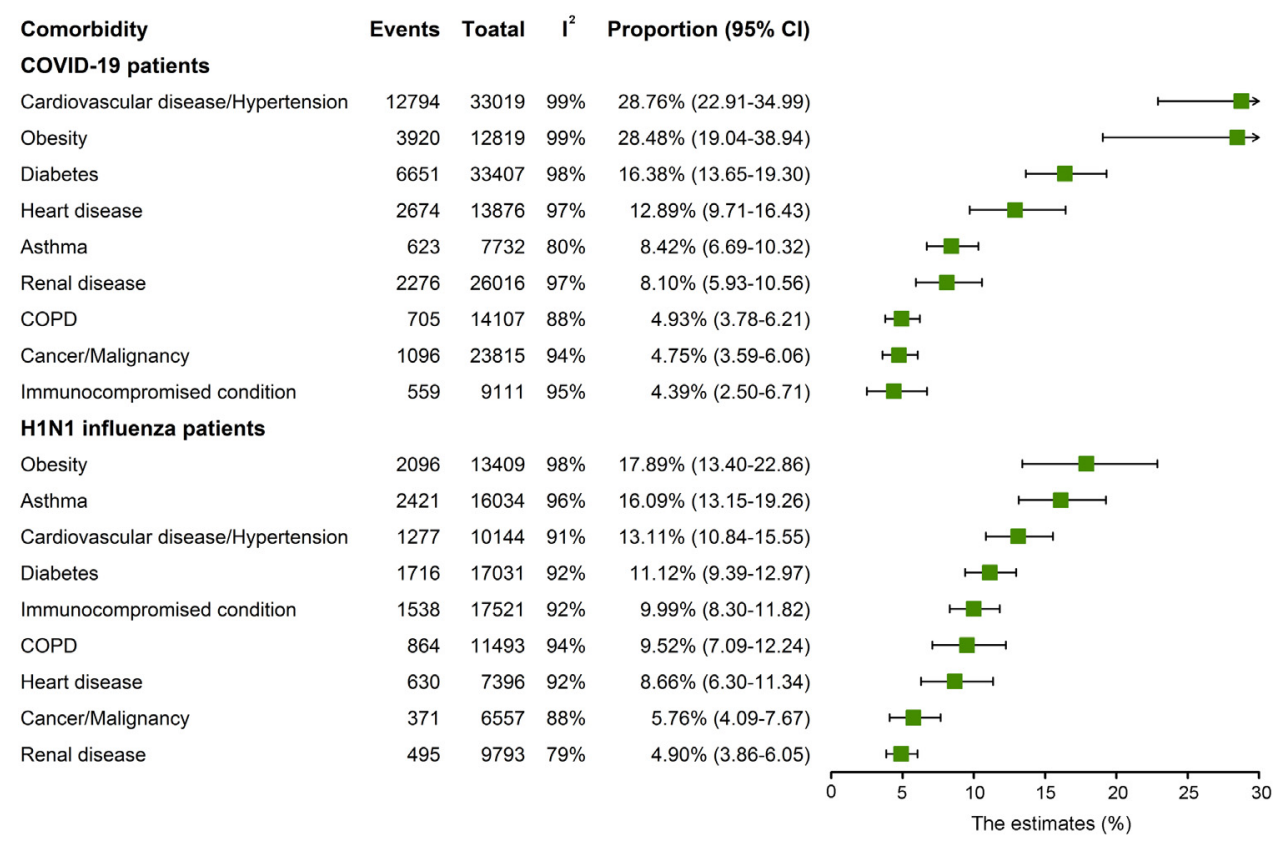

Figure 3. Forest plot of incidence rate of comorbidities in hospitalized COVID-19 and 2009 influenza patients. 
immunocompromised conditions (9.99\%, 95\% CI 8.30-11.82; $I^{2}=$ $92 \%)$, chronic obstructive pulmonary disease (COPD) $(9.52 \%, 95 \% \mathrm{CI}$ $\left.7.09-12.24 ; I^{2}=94 \%\right)$, heart disease $\left(8.66 \%, 95 \%\right.$ CI $6.30-11.34 ; I^{2}=$ $92 \%)$, cancer/malignancy $\left(5.76 \%, 95 \%\right.$ CI $\left.4.09-7.67 ; I^{2}=88 \%\right)$ and renal disease $\left(4.90 \%, 95 \%\right.$ CI $3.86-6.05 \% ; I^{2}=79 \%$ ) (Figure 3 ; Supplementary data S30-38). Importantly, the estimated incidence rates of cardiovascular disease/hypertension $(28.76 \%$ vs. $13.11 \%$ ) and diabetes (16.38\% vs. $11.12 \%$ ) were significantly higher in COVID-19 compared with influenza patients. In contrast, comorbidities such as asthma (16.09\% vs. $8.42 \%$ ), COPD (9.52\% vs. $4.93 \%)$ and immunocompromised conditions (9.99\% vs. $4.39 \%$ ) were significantly more common in influenza patients compared with COVID-19 patients ( $p<0.05$; Table 2$)$.

Comparative analysis of clinical management and outcome between hospitalized COVID-19 and influenza patients

Clinical management for hospitalized COVID-19 and influenza patients was comparatively analyzed. Because secondary bacterial infections are common after viral infection, antibiotics were widely used in these patients. It was found that the frequency of antibiotic treatment was similar between hospitalized COVID-19 (67.44\%, 95\% CI 51.91-81.27; $\left.I^{2}=99 \%\right)$ and H1N1 (60.45\%, 95\% CI 47.93-72.31; $I^{2}=97 \%$ ) patients (Table 1). Unexpectedly, the estimated rates of intensive care unit (ICU) admissions (17.74\%, 95\% CI 13.93-21.89 vs. $16.03 \%$, 95\% CI 11.52-21.06) and treatment with extracorporeal membrane oxygenation (ECMO) $(3.10 \%, 95 \% \mathrm{CI}$ $1.45-5.25$ vs. $5.79 \%$ ) were comparable between hospitalized COVID-19 and 2009 influenza pandemic patients. Treatment with mechanical ventilation appeared to be more common in COVID-19 $\left(27.10 \%, 95 \%\right.$ CI $\left.20.91-33.75 ; I^{2}=99 \%\right)$ than influenza $(14.70 \%, 95 \%$ CI $\left.6.65 \%-25.12 \% ; I^{2}=99 \%\right)$ patients, although this was not statistically significant. Most surprisingly, the fatality rate of hospitalized patients was slightly higher in COVID-19 (12.94\%, 95\% CI 10.93-15.10; $\left.I^{2}=95 \%\right)$ than 2009 influenza patients $(9.63 \%, 95 \%$ CI 6.05-13.90; $I^{2}=95 \%$ ) (Table 1 ; Supplementary data 39-47).

\section{Discussion}

This study provided a comprehensive comparison of hospitalized patients between the 2009 influenza and COVID-19 pandemics, regarding demographics, symptoms, comorbidity, management, and outcomes. Hospitalized COVID-19 patients compared with influenza patients were much older. This supports the notion that SARS-CoV-2 primarily causes severe diseases in the elderly (Verity et al., 2020), whereas H1N1 influenza severely affected younger populations (Reed et al., 2014; Viboud and Simonsen, 2012). Besides the common symptoms of respiratory infections, it was found that a proportion of patients from both pandemics had diarrhea and vomiting/nausea symptoms. Both SARS-CoV-2 and influenza viruses have been shown to effectively infect and replicate in the intestinal epithelium (Qu et al., 2012; Xiao et al., 2020), causing gastrointestinal symptoms in patients (Gu et al., 2020; Patel et al., 2010). Consistent with previous studies reporting cardiovascular disease, hypertension and diabetes as risk factors of COVID-19 (Zhou et al., 2020), a substantially high incidence rate of these comorbidities was found in COVID-19 patients.

It was clear that the severity and mortality of SARS-CoV-2 infection were much higher than those of the influenza infection in the general population (Ji et al., 2020; Petersen et al., 2020). This study revealed a completely different picture in the hospitalized patient population. It found a $>15 \%$ incidence rate of inpatients admitted to ICU in both H1N1 influenza and COVID-19 pandemics, suggesting comparable severity of disease prognosis in these hospitalized patients. Hospitalized COVID-19 and 2009 influenza patients consistently had similar death rates (12.94\% vs. $9.63 \%)$.

This study focused on comparing COVID-19 patients with 2009 pandemic influenza H1N1 patients; however, it would also be interesting to compare with seasonal flu caused by influenza $A$ and $\mathrm{B}$ viruses. Hospitalized pandemic H1N1 patients in this analysis appeared to have higher frequencies of fever, fatigue, vomiting, shortness of breath, and diarrhea compared with hospitalized seasonal influenza patients (Zhang et al., 2020). However, comorbidities - including diabetes, COPD, immunocompromised status, and heart disease - seemed more common in seasonal influenza patients compared with $\mathrm{H} 1 \mathrm{~N} 1$ pandemic patients (Mohammad et al., 2019). Nevertheless, future studies are required to comprehensively, for example by systematic review and metaanalysis, compare COVID-19 with pandemic and seasonal influenza.

There has been recent attention on comparing COVID-19 with influenza or the infection of classical endemic coronaviruses in pediatric populations. A comparison between children with COVID-19 and seasonal influenza showed no differences in hospitalization rates, ICU admission rates, and mechanical ventilator use (Song et al., 2020). In infants aged $<1$ year, major differences in the clinical features are unlikely when comparing SARS-CoV-2 with classical endemic coronaviruses or influenza virus symptoms (Li et al., 2020a, 2020b; Vanhems et al., 2020). Interestingly, some studies suggest that clinical manifestations of COVID-19 are even milder than influenza $A$ in children aged $<5$ years (Li et al., 2020c).

This study had some limitations. First, the criteria for hospitalization may have differed across different studies, resulting in patient selection bias. Second, it was unable to estimate the incidence rate of ECMO treatment for 2009 influenza patients because only two studies were available. Third, the number of available studies on the 2009 influenza pandemic was limited, which may have caused estimation bias. Finally, the COVID-19 pandemic is still ongoing and evolving. Future studies may update the current estimations on the clinical features of hospitalized COVID-19 patients.

In summary, this meta-analysis comprehensively deciphered the clinical features for hospitalized patients comparing COVID-19 with the 2009 influenza pandemic. Similar levels of disease burden were found in hospitalized COVID-19 and influenza patients from these two pandemics. This is in contrast with the assumption for the general population that COVID-19 causes much more severe damage compared to influenza. Thus, the devastating situation of COVID-19 could be mainly attributed to the rapid spread and it affecting a large population, whereas the spread of the 2009 pandemic influenza was relatively limited and affected a smaller population.

\section{Funding}

This research was supported by the Major National Special Funds for Science and Technology (2015ZX09102016) and the Changjiang Scholars and Innovative Research Team in University grant (No. IRT_17R88) from the Ministry of Education of the People's Republic of China to Z. Ma, and a VIDI grant (No. 91719300) from theNetherlands Organisation for Scientific Research (NWO) to Q. Pan,

\section{Authors contributions}

P.L., M.P.P., Z.M., and Q.P. conceived the idea and interpreted data. P.L., Y.W. and Q.P. conducted data analysis. P.L. wrote the manuscript. Q.P. critically revised the manuscript. 


\section{Conflict of interest}

All the authors declare that they have no conflict of interest.

\section{Acknowledgements}

We greatly thank Maarten F.M. Engel from the Medical Library, Erasmus MC-University Medical Center for conducting the literature search.

\section{Appendix A. Supplementary data}

Supplementary material related to this article can be found, in the online version, at doi:https://doi.org/10.1016/j.ijid.2020.11.127.

\section{References}

Adhikari SP, Meng S, Wu YJ, Mao YP, Ye RX, Wang QZ, et al. Epidemiology, causes, clinical manifestation and diagnosis, prevention and control of coronavirus disease (COVID-19) during the early outbreak period: a scoping review. Infect Dis Poverty 2020;9(1):29.

Gu J, Han B, Wang J. COVID-19: Gastrointestinal Manifestations and Potential FecalOral Transmission. Gastroenterology 2020;158(6):1518-9.

He D, Zhao S, Li Y, Cao P, Gao D, Lou Y, et al. Comparing COVID-19 and the 1918-19 influenza pandemics in the United Kingdom. Int J Infect Dis 2020;98:67-70.

Huang C, Wang Y, Li X, Ren L, Zhao J, Hu Y, et al. Clinical features of patients infected with 2019 novel coronavirus in Wuhan, China. Lancet 2020;395(10223):497506.

Ji Y, Ma Z, Peppelenbosch MP, Pan Q. Potential association between COVID-19 mortality and health-care resource availability. Lancet Glob Health 2020;8(4): e480.

Li P, Ikram A, Peppelenbosch MP, Ma Z, Pan Q. Systematically mapping clinical features of infections with classical endemic human coronaviruses. Clin Infect Dis 2020a;

Li P, Liu J, Ma Z, Bramer WM, Peppelenbosch MP, Pan Q. Estimating Global Epidemiology of Low-Pathogenic Human Coronaviruses in Relation to the COVID-19 Context. J Infect Dis 2020b;222(4):695-6.

Li Y, Wang H, Wang F, Du H, Liu X, Chen P, et al. Comparison of hospitalized patients with pneumonia caused by COVID-19 and influenza A in children under 5 years. Int J Infect Dis 2020c;98:80-3.
Mohammad S, Korn K, Schellhaas B, Neurath MF, Goertz RS. Clinical Characteristics of Influenza in Season 2017/2018 in a German Emergency Department: A Retrospective Analysis. Microbiol Insights 2019;12:1178636119890302.

Munn Z, Moola S, Lisy K, Riitano D, Tufanaru C. Chapter 5: Systematic reviews of prevalence and incidence. In: Aromataris E, Munn Z, editors. JBI Manual for Evidence Synthesis. JBI; 2020, doi:http://dx.doi.org/10.46658/JBIMES-20-06 Available from https://synthesismanual.jbi.global..

Patel M, Dennis A, Flutter C, Khan Z. Pandemic (H1N1) 2009 influenza. Br J Anaesth 2010;104(2):128-42.

Petersen E, Koopmans M, Go U, Hamer DH, Petrosillo N, Castelli F, et al. Comparing SARS-CoV-2 with SARS-CoV and influenza pandemics. Lancet Infect Dis 2020;

Petrosillo N, Viceconte G, Ergonul O, Ippolito G, Petersen E. COVID-19, SARS and MERS: are they closely related?. Clin Microbiol Infect 2020;26(6):729-34.

Qu B, Li X, Gao W, Sun W, Jin Y, Cardona CJ, et al. Human intestinal epithelial cells are susceptible to influenza virus subtype H9N2. Virus Res 2012;163(1):151-9.

Reed C, Chaves SS, Perez A, D'Mello T, Daily Kirley P, Aragon D, et al. Complications among adults hospitalized with influenza: a comparison of seasonal influenza and the 2009 H1N1 pandemic. Clin Infect Dis 2014:59(2):166-74.

Rothan HA, Byrareddy SN. The epidemiology and pathogenesis of coronavirus disease (COVID-19) outbreak. J Autoimmun 2020;109:102433.

Song X, Delaney M, Shah RK, Campos JM, Wessel DL, DeBiasi RL. Comparison of Clinical Features of COVID-19 vs Seasonal Influenza A and B in US Children. JAMA Netw Open 2020;3(9)e2020495.

Vanhems P, Endtz H, Dananche C, Komurian-Pradel F, Sanchez Picot V. Pneumonia Study Gm. Comparison of the Clinical Features of SARS-CoV-2, Other Coronavirus and Influenza Infections in Infants Less Than 1-Year-Old. Pediatr Infect Dis J 2020;39(7) e157-e8.

Verity R, Okell LC, Dorigatti I, Winskill P, Whittaker C, Imai N, et al. Estimates of the severity of coronavirus disease 2019: a model-based analysis. Lancet Infect Dis 2020;20(6):669-77.

Viboud C, Simonsen L. Global mortality of 2009 pandemic influenza A H1N1. Lancet Infect Dis 2012:12(9):651-3.

Xiao F, Tang M, Zheng X, Liu Y, Li X, Shan H. Evidence for Gastrointestinal Infection of SARS-CoV-2. Gastroenterology 2020;158(6) 1831-3 e3.

Zhang J, Ding D, Huang X, Zhang J, Chen D, Fu P, et al. Differentiation of COVID-19 from seasonal influenza: a multicenter comparative study. J Med Virol 2020;

Zhao W, Zhong Z, Xie X, Yu Q, Liu J. Relation Between Chest CT Findings and Clinical Conditions of Coronavirus Disease (COVID-19) Pneumonia: a Multicenter Study. AJR Am J Roentgenol 2020;214(5):1072-7.

Zhou F, Yu T, Du R, Fan G, Liu Y, Liu Z, et al. Clinical course and risk factors for mortality of adult inpatients with COVID-19 in Wuhan, China: a retrospective cohort study. Lancet 2020;395(10229):1054-62. 